\title{
On the solvability of one boundary value problem for some semilinear wave equations with source terms
}

Sergo Kharibegashvili and Bidzina Midodashvili

\begin{abstract}
In a conic domain of time type for one class of semilinear wave equations with source terms we consider a Sobolev problem representing a multidimensional version of the Darboux second problem. The questions on global and local solvability, uniqueness and absence of solutions of this problem are investigated.
\end{abstract}

Mathematics Subject Classification (2010). 35L05, 35L70.

Keywords. Sobolev problem, Semilinear wave equations, Source terms, Global and local solvability, Nonexistence.

\section{Introduction}

Consider a semilinear wave equation of the form

$$
L_{\lambda} u:=\frac{\partial^{2} u}{\partial t^{2}}-\triangle u+\lambda f(u)=F,
$$

where $\lambda \neq 0$ is a given real constant, $f$ and $F$ are given real functions, besides, $f$ is a nonlinear one and $u$ is an unknown real function, $\triangle=\sum_{i=1}^{n} \frac{\partial^{2}}{\partial x_{i}^{2}}, n \geq 2$.

Let $D$ be a conic domain in the space $\mathbb{R}^{n+1}$ of the variables $x=\left(x_{1}, x_{2}\right.$, $\left.\ldots, x_{n}\right)$ and $t$, i.e. $D$ contains, along with the point $(x, t) \in D$, the whole ray $l:(\tau x, \tau t), 0<\tau<\infty$. Denote by $S$ the conic surface of $\partial D$. Suppose that $D$ is homeomorphic to the conic domain $\omega: t>|x|$, and $S \backslash O$ is a connected $n$-dimensional manifold of the class $C^{\infty}$, where $O=(0, \ldots, 0,0)$ is the vertex of $S$. Suppose also that $D$ lies in the half-space $t>0$ and $D_{T}:=\{(x, t) \in D$ : $t<T\}, S_{T}:=\{(x, t) \in S: t \leq T\}, T>0$. It is clear that if $T=\infty$, then $D_{\infty}=D$ and $S_{\infty}=S$. 
For the equation (1.1) we consider the problem on finding of a solution $u(x, t)$ of that equation in the domain $D_{T}$ by the boundary condition

$$
\left.u\right|_{S_{T}}=g,
$$

where $g$ is a given real function on $S_{T}$.

In the linear case, when $\lambda=0$ and the conic manifold $S=\partial D$ is timeoriented, i.e.

$$
\left.\left(\nu_{0}^{2}-\sum_{i=1}^{n} \nu_{i}^{2}\right)\right|_{S}<0,\left.\quad \nu_{0}\right|_{S}<0,
$$

where $\nu=\left(\nu_{1}, \ldots, \nu_{n}, \nu_{0}\right)$ is the unit vector of the outer normal to $S \backslash O$, the problem (1.1), (1.2) was posed by S.L. Sobolev in the work [1], where the unique solvability of this problem in the corresponding functional spaces is given. In the same work [1] it is made a conjecture that the obtained results will be valid for nonlinear wave equations. The aim of the present paper is to reveal conditions for nonlinear source term in the equation (1.1) which will ensure a global solvability of the problem (1.1), (1.2) in some certain cases and be a cause of nonexistence of a global solution of this problem in other cases, although in all these cases the considering problem will be locally solvable. In the particular case of nonlinearity when $f(u)=|u|^{p} u, p=$ const $>0$ and the homogeneous boundary condition (1.2), i.e. for $g=0$, the problem (1.1), (1.2) has been considered in [2], while for the nonhomogeneous boundary condition (1.2) this problem has been studied in [3]. Note that in the case (1.3) the problem (1.1), (1.2) can be considered as a multidimensional version of the Darboux second problem [4, pp. 228, 233] for the semilinear equation (1.1).

It should be noted also that for the nonlinear wave equations (1.1) other problems (Cauchy problem with initial conditions at $t=0$, Cauchy characteristic problem in the light cone of future, mixed and periodical problems) are considered in numerous literature [see, e.g., [5-24]].

Below we require that the condition (1.3) is fulfilled and the nonlinear function $f$ satisfies the following requirement

$$
f \in C(\mathbb{R}), \quad|f(u)| \leq M_{1}+M_{2}|u|^{\alpha}, \quad \alpha=\text { const }>0, \quad u \in \mathbb{R},
$$

besides, in the following sections we consider also the condition

$$
\int_{0}^{u} f(s) d s \geq-M_{3}-M_{4} u^{2}, \quad u \in \mathbb{R},
$$

where $M_{i}=$ const $\geq 0, i=1,2,3,4$.

Remark 1.1. In the case when $\alpha \leq 1$ from the inequality (1.4) it follows the inequality (1.5). It is obvious also that the function $f(u)=|u|^{\alpha}$ signu satisfies both conditions (1.4) and (1.5) for $M_{1}=0, M_{2}=1, M_{3}=M_{4}=0$, since $\int_{0}^{u}|s|^{\alpha}$ signsds $=\frac{1}{\alpha+1}|u|^{\alpha+1}, \alpha>0$.

Denote by $W_{2}^{k}(\Omega)$ the Sobolev space consisting of the elements of $L_{2}(\Omega)$, having in $L_{2}(\Omega)$ all generalized derivatives up to the $k$ th order, inclusively. 
Remark 1.2. The embedding operator $I: W_{2}^{1}\left(D_{T}\right) \rightarrow L_{q}\left(D_{T}\right)$ is the linear continuous compact operator for $1<q<\frac{2(n+1)}{n-1}$, when $n>1$ [25, p. 86]. At the same time the Nemitsky operator $K: L_{q}\left(D_{T}\right) \rightarrow L_{2}\left(D_{T}\right)$, acting according to the formula $K u=f(u)$, where the function $f$ satisfies the condition (1.4), is continuous and bounded for $q \geq 2 \alpha$ [26, p. 349], [27, pp. 66, 67]. Thus, if $\alpha<\frac{n+1}{n-1}$, i.e. $2 \alpha<\frac{2(n+1)}{n-1}$, then there exists a number $q$ such that $1<q<\frac{2(n+1)}{n-1}$ and $q \geq 2 \alpha$. Therefore, in this case the operator

$$
K_{0}=K I: W_{2}^{1}\left(D_{T}\right) \rightarrow L_{2}\left(D_{T}\right)
$$

is continuous and compact. Moreover, $f(u) \in L_{2}\left(D_{T}\right)$ follows from $u \in W_{2}^{1}$ $\left(D_{T}\right)$, and if $u_{m} \rightarrow u$ in the space $W_{2}^{1}\left(D_{T}\right)$, then $f\left(u_{m}\right) \rightarrow f(u)$ in the space $L_{2}\left(D_{T}\right)$.

Definition 1.3. Let $F \in L_{2}\left(D_{T}\right), g \in W_{2}^{1}\left(S_{T}\right)$ and the condition (1.4) be fulfilled with $0<\alpha<\frac{n+1}{n-1}$. We call a function $u \in W_{2}^{1}\left(D_{T}\right)$ a strong generalized solution of the nonlinear problem (1.1), (1.2) of the class $W_{2}^{1}$ in the domain $D_{T}$ if there exists a sequence of functions $u_{k} \in C^{2}\left(\bar{D}_{T}\right)$ such that $u_{k} \rightarrow u$ in the space $W_{2}^{1}\left(D_{T}\right), L_{\lambda} u_{k} \rightarrow F$ in the space $L_{2}\left(D_{T}\right)$ and $\left.u_{k}\right|_{S_{T}} \rightarrow g$ in the space $W_{2}^{1}\left(S_{T}\right)$. The convergence of the sequence $\left\{\lambda f\left(u_{k}\right)\right\}$ to function $\lambda f(u)$ in the space $L_{2}\left(D_{T}\right)$ when $u_{k} \rightarrow u$ in the space $W_{2}^{1}\left(D_{T}\right)$ follows from Remark 1.2.

Definition 1.4. Let $0<\alpha<\frac{n+1}{n-1}$, the condition (1.4) be fulfilled and $F \in$ $L_{2, l o c}(D), g \in W_{2, l o c}^{1}(S)$ and $\left.F\right|_{D_{T}} \in L_{2}\left(D_{T}\right),\left.g\right|_{S_{T}} \in W_{2}^{1}\left(S_{T}\right)$ for every $T>0$. We say that the problem (1.1), (1.2) is globally solvable in the class $W_{2}^{1}$ if for every $T>0$ this problem has a strong generalized solution of the class $W_{2}^{1}$ in the domain $D_{T}$.

Definition 1.5. Let $0<\alpha<\frac{n+1}{n-1}$, the condition (1.4) be fulfilled and $F \in$ $L_{2, l o c}(D), g \in W_{2, l o c}^{1}(S)$ and $\left.F\right|_{D_{T}} \in L_{2}\left(D_{T}\right),\left.g\right|_{S_{T}} \in W_{2}^{1}\left(S_{T}\right)$ for every $T>0$. We say that the problem (1.1), (1.2) is locally solvable in the class $W_{2}^{1}$ if there exists a number $T_{0}=T_{0}(F, g)>0$ such that for $T<T_{0}$ this problem has a strong generalized solution of the class $W_{2}^{1}$ in the domain $D_{T}$.

The work is organized as follows. In Sect. 2 it is received a priori estimate of the solution of the problem (1.1), (1.2) of the class $W_{2}^{1}$, which is used in the proof of the global solvability of this problem in Sect. 3. In Sect. 4, under certain requirements on the nonlinear function $f$, it is proved the uniqueness of the solution of the problem (1.1), (1.2) of the class $W_{2}^{1}$ (this question has not been considered in $[2,3])$. In Sect. 5 we consider the cases of absence of global solvability and in the last section it is established the local solvability of the problem (1.1), (1.2).

\section{A priori estimate of the solution of the problem (1.1), (1.2) of the class $W_{2}^{1}$}

Lemma 2.1. Let $\lambda>0$, the conditions (1.3), (1.4), (1.5) be fulfilled, $0<\alpha<$ $\frac{n+1}{n-1}, F \in L_{2}\left(D_{T}\right)$ and $g \in W_{2}^{1}\left(S_{T}\right)$. Then for every strong generalized solution 
$u$ of the problem (1.1), (1.2) of the class $W_{2}^{1}$ in the domain $D_{T}$ the a priori estimate

$$
\|u\|_{W_{2}^{1}\left(D_{T}\right)} \leq c_{1}\|F\|_{L_{2}\left(D_{T}\right)}+c_{2}\|g\|_{W_{2}^{1}\left(S_{T}\right)}+c_{3}\|g\|_{W_{2}^{1}\left(S_{T}\right)}^{\frac{1}{2}}+c_{4}
$$

is valid with nonnegative constants $c_{i}=c_{i}(\lambda, S, f, T), 1 \leq i \leq 4$, not depending on $u, g$ and $F$, where $c_{j}>0, j=1,2$.

Proof. Let $u \in W_{2}^{1}\left(D_{T}\right)$ be a strong generalized solution of the problem (1.1), (1.2) of the class $W_{2}^{1}$ in the domain $D_{T}$. Then, in view of Definition 1.3, there exists the sequence of functions $u_{k} \in C^{2}\left(\bar{D}_{T}\right)$ such that

$$
\begin{aligned}
& \lim _{k \rightarrow \infty}\left\|u_{k}-u\right\|_{W_{2}^{1}\left(D_{T}\right)}=0, \quad \lim _{k \rightarrow \infty}\left\|L_{\lambda} u_{k}-F\right\|_{L_{2}\left(D_{T}\right)}=0, \\
& \lim _{k \rightarrow \infty}\left\|\left.u_{k}\right|_{S_{T}}-g\right\|_{W_{2}^{1}\left(S_{T}\right)}=0 .
\end{aligned}
$$

Consider the function $u_{k} \in C^{2}\left(\bar{D}_{T}\right)$ as a solution of the following problem

$$
\begin{aligned}
& L_{\lambda} u_{k}=F_{k}, \\
& \left.u_{k}\right|_{S_{T}}=g_{k} .
\end{aligned}
$$

Here

$$
F_{k}:=L_{\lambda} u_{k}, \quad g_{k}:=\left.u_{k}\right|_{S_{T}} .
$$

Putting

$$
\zeta(u):=\int_{0}^{u} f(s) d s
$$

and multiplying the both sides of the equation (2.4) by $\frac{\partial u_{k}}{\partial t}$, after integration in the domain $D_{\tau}:=\{(x, t) \in D: t<\tau\}, 0<\tau \leq T$, we receive

$$
\begin{array}{r}
\frac{1}{2} \int_{D_{\tau}} \frac{\partial}{\partial t}\left(\frac{\partial u_{k}}{\partial t}\right)^{2} d x d t-\int_{D_{\tau}} \triangle u_{k} \frac{\partial u_{k}}{\partial t} d x d t \\
+\lambda \int_{D_{\tau}} \frac{\partial}{\partial t} \zeta\left(u_{k}\right) d x d t=\int_{D_{\tau}} F_{k} \frac{\partial u_{k}}{\partial t} d x d t .
\end{array}
$$

Let $\Omega_{\tau}:=D \cap\{t=\tau\}$ and denote by $\nu=\left(\nu_{1}, \ldots, \nu_{n}, \nu_{0}\right)$ the unit vector of the outer normal to $S_{T} \backslash\{(0, \ldots, 0,0)\}$. Integrating by parts and taking into account the equalities $(2.5)$ and $\left.\nu\right|_{\Omega_{\tau}}=(0, \ldots, 0,1)$ we receive

$$
\begin{aligned}
& \int_{D_{\tau}} \frac{\partial}{\partial t}\left(\frac{\partial u_{k}}{\partial t}\right)^{2} d x d t=\int_{\partial D_{\tau}}\left(\frac{\partial u_{k}}{\partial t}\right) 2 \nu_{0} d s=\int_{\Omega_{\tau}}\left(\frac{\partial u_{k}}{\partial t}\right) 2 d x+\int_{S_{\tau}}\left(\frac{\partial u_{k}}{\partial t}\right) 2 \nu_{0} d s, \\
& \lambda \int_{D_{\tau}} \frac{\partial}{\partial t} \zeta\left(u_{k}\right) d x d t=\lambda \int_{\partial D_{\tau}} \zeta\left(u_{k}\right) \nu_{0} d s=\lambda \int_{S_{\tau}} \zeta\left(g_{k}\right) \nu_{0} d s+\lambda \int_{\Omega_{\tau}} \zeta\left(u_{k}\right) d x,
\end{aligned}
$$




$$
\begin{aligned}
\int_{D_{\tau}} & \frac{\partial^{2} u_{k}}{\partial x_{i}^{2}} \frac{\partial u_{k}}{\partial t} d x d t=\int_{\partial D_{\tau}} \frac{\partial u_{k}}{\partial x_{i}} \frac{\partial u_{k}}{\partial t} \nu_{i} d s-\frac{1}{2} \int_{D_{\tau}} \frac{\partial}{\partial t}\left(\frac{\partial u_{k}}{\partial x_{i}}\right)^{2} d x d t \\
= & \int_{\partial D_{\tau}} \frac{\partial u_{k}}{\partial x_{i}} \frac{\partial u_{k}}{\partial t} \nu_{i} d s-\frac{1}{2} \int_{\partial D_{\tau}}\left(\frac{\partial u_{k}}{\partial x_{i}}\right)^{2} \nu_{0} d s=\int_{\partial D_{\tau}} \frac{\partial u_{k}}{\partial x_{i}} \frac{\partial u_{k}}{\partial t} \nu_{i} d s \\
& -\frac{1}{2} \int_{S_{\tau}}\left(\frac{\partial u_{k}}{\partial x_{i}}\right)^{2} \nu_{0} d s-\frac{1}{2} \int_{\Omega_{\tau}}\left(\frac{\partial u_{k}}{\partial x_{i}}\right)^{2} d x
\end{aligned}
$$

Whence due to $(2.8)$ it follows that

$$
\begin{aligned}
\int_{D_{\tau}} & F_{k} \frac{\partial u_{k}}{\partial t} d x d t=\int_{S_{\tau}} \frac{1}{2 \nu_{0}}\left[\sum_{i=1}^{n}\left(\frac{\partial u_{k}}{\partial x_{i}} \nu_{0}-\frac{\partial u_{k}}{\partial t} \nu_{i}\right)^{2}\right. \\
& \left.+\left(\frac{\partial u_{k}}{\partial t}\right)^{2}\left(\nu_{0}^{2}-\sum_{j=1}^{n} \nu_{j}^{2}\right)\right] d s+\frac{1}{2} \int_{\Omega_{\tau}}\left[\left(\frac{\partial u_{k}}{\partial t}\right)^{2}+\sum_{i=1}^{n}\left(\frac{\partial u_{k}}{\partial x_{i}}\right)^{2}\right] d x \\
& +\lambda \int_{S_{\tau}} \zeta\left(g_{k}\right) \nu_{0} d s+\lambda \int_{\Omega_{\tau}} \zeta\left(u_{k}\right) d x
\end{aligned}
$$

Since $\lambda>0$ and in view of (1.3), (1.5) and (2.7) from (2.9) it follows that

$$
\begin{aligned}
& \frac{1}{2} \int_{\Omega_{\tau}}\left[\left(\frac{\partial u_{k}}{\partial t}\right)^{2}+\sum_{i=1}^{n}\left(\frac{\partial u_{k}}{\partial x_{i}}\right)^{2}\right] d x \leq \int_{S_{\tau}} \frac{1}{2\left|\nu_{0}\right|}\left[\sum_{i=1}^{n}\left(\frac{\partial u_{k}}{\partial x_{i}} \nu_{0}-\frac{\partial u_{k}}{\partial t} \nu_{i}\right)^{2}\right] d s \\
& \quad+\lambda \int_{S_{\tau}}\left|\zeta\left(g_{k}\right)\right|\left|\nu_{0}\right| d s+\lambda \int_{\Omega_{\tau}}\left[M_{3}+M_{4} u_{k}^{2}\right] d x+\int_{D_{\tau}} F_{k} \frac{\partial u_{k}}{\partial t} d x d t .
\end{aligned}
$$

Because $S$ represents a conic manifold, then $\sup _{S \backslash O}\left|\nu_{0}\right|^{-1}=\sup _{S \cap\{t=1\}}$ $\left|\nu_{0}\right|^{-1}$. At the same time $S \backslash O$ represents a smooth manifold and $S \cap\{t=1\}=$ $\partial \Omega_{\tau=1}$ is a compact manifold. Therefore, since $\nu_{0}$ is the continuous function on $S \backslash O$, we have

$$
M_{0}:=\sup _{S \backslash O}\left|\nu_{0}\right|^{-1}=\sup _{S \cap\{t=1\}}\left|\nu_{0}\right|^{-1}<+\infty, \quad\left|\nu_{0}\right| \leq|\nu|=1 .
$$

Noting that $\left(\nu_{0} \frac{\partial}{\partial x_{i}}-\nu_{i} \frac{\partial}{\partial t}\right), i=1, \ldots, n$, is an inner differential operator on $S_{T}$ and due to (2.5) we obtain

$$
\int_{S_{\tau}}\left[\sum_{i=1}^{n}\left(\frac{\partial u_{k}}{\partial x_{i}} \nu_{0}-\frac{\partial u_{k}}{\partial t} \nu_{i}\right)^{2}\right] d s \leq\left\|\left.u_{k}\right|_{S_{T}}\right\|_{W_{2}^{1}\left(S_{T}\right)}^{2}=\left\|g_{k}\right\|_{W_{2}^{1}\left(S_{T}\right)}^{2} .
$$

From (2.11), (2.12) it follows that

$$
\int_{S_{\tau}} \frac{1}{2\left|\nu_{0}\right|}\left[\sum_{i=1}^{n}\left(\frac{\partial u_{k}}{\partial x_{i}} \nu_{0}-\frac{\partial u_{k}}{\partial t} \nu_{i}\right)^{2}\right] d s \leq \frac{1}{2} M_{0}\left\|g_{k}\right\|_{W_{2}^{1}\left(S_{T}\right)}^{2} .
$$

In view of (1.4) and (2.7) we have

$$
|\zeta(u)|=\left|\int_{0}^{u} f(s) d s\right| \leq M_{1}|u|+\frac{M_{2}}{\alpha+1}|u|^{\alpha+1} .
$$


By virtue of the Cauchy inequality $2 F_{k} \frac{\partial u_{k}}{\partial t} \leq F_{k}^{2}+\left(\frac{\partial u_{k}}{\partial t}\right)^{2}$ and due to (2.13), (2.14) from (2.10) we have

$$
\begin{aligned}
\int_{\Omega_{\tau}} & {\left[\left(\frac{\partial u_{k}}{\partial t}\right)^{2}+\sum_{i=1}^{n}\left(\frac{\partial u_{k}}{\partial x_{i}}\right)^{2}\right] d x \leq M_{0}|| g_{k} \|_{W_{2}^{1}\left(S_{T}\right)}^{2}+2 \lambda M_{1} \int_{S_{\tau}}\left|g_{k}\right| d s } \\
& +\frac{2 \lambda M_{2}}{\alpha+1} \int_{S_{\tau}}\left|g_{k}\right|^{\alpha+1} d s+2 \lambda M_{3} m e s \Omega_{\tau}+2 \lambda M_{4} \int_{\Omega_{\tau}} u_{k}^{2} d x \\
& +\int_{D_{T}}\left(\frac{\partial u_{k}}{\partial t}\right)^{2} d x d t+\int_{D_{T}} F_{k}^{2} d x d t, 0<\tau \leq T .
\end{aligned}
$$

Further, according to the Schwartz inequality we have

$$
\begin{aligned}
& \int_{S_{\tau}}\left|g_{k}\right| d s \leq\left(\int_{S_{\tau}} 1^{2} d s\right)^{\frac{1}{2}}\left(\int_{S_{\tau}}\left|g_{k}\right|^{2} d s\right)^{\frac{1}{2}} \\
& \quad \leq\left(\operatorname{mes} S_{\tau}\right)^{\frac{1}{2}}\left\|g_{k}\right\|_{W_{2}^{1}\left(S_{\tau}\right)} \leq\left(\operatorname{mes} S_{T}\right)^{\frac{1}{2}}\left\|g_{k}\right\|_{W_{2}^{1}\left(S_{T}\right)} .
\end{aligned}
$$

Considerations from Remark 1.2 regarding to the space $W_{2}^{1}\left(S_{T}\right)$, taking into account that $\operatorname{dim} S_{T}=\operatorname{dim} D_{T}-1=n$, show that embedding operator $I: W_{2}^{1}\left(S_{T}\right) \rightarrow L_{q}\left(S_{T}\right)$ is a linear continuous compact operator for $1<$ $q<\frac{2 n}{n-2}, n \geq 2\left[25\right.$, p. 86]. At the same time the Nemitsky operator $K_{1}$ : $L_{q}\left(S_{T}\right) \rightarrow L_{2}\left(S_{T}\right)$, acting according to the formula $K_{1} u=|u|^{\frac{\alpha+1}{2}}$ is continuous and bounded for $q \geq 2 \frac{\alpha+1}{2}=\alpha+1$ [26, p. 349], [27, pp. 66, 67]. Thus, if $\alpha+1<\frac{2 n}{n-2}$, i.e. $\alpha<\frac{n+2}{n-2}$, then there exists number $q$, such that $1<q<\frac{2 n}{n-2}$ and $q \geq \alpha+1$. Therefore in this case the operator

$$
K_{2}=K_{1} I: W_{2}^{1}\left(S_{T}\right) \rightarrow L_{2}\left(S_{T}\right)
$$

will be continuous and compact. Hence, according to the inequality $\frac{n+1}{n-1}<\frac{n+2}{n-2}$ and $(2.3),(2.5)$, it follows that

$$
\lim _{k \rightarrow \infty} \int_{S_{\tau}}\left|g_{k}\right|^{\alpha+1} d s=\int_{S_{\tau}}|g|^{\alpha+1} d s
$$

and

$$
\int_{S_{T}}|g|^{\alpha+1} d s \leq C_{1}\|g\|_{W_{2}^{1}\left(S_{T}\right)}^{2}
$$

with a positive constant $C_{1}=C_{1}(T)$, not depending on $g \in W_{2}^{1}\left(S_{T}\right)$.

If $t=\gamma(x)$ represents an equation of the conic surface $S$, then due to (2.5) we have

$$
\begin{aligned}
u_{k}(x, \tau)= & u_{k}(x, \gamma(x))+\int_{\gamma(x)}^{\tau} \frac{\partial}{\partial t} u_{k}(x, s) d s=g_{k}(x) \\
& +\int_{\gamma(x)}^{\tau} \frac{\partial}{\partial t} u_{k}(x, s) d s,(x, \tau) \in \Omega_{\tau}
\end{aligned}
$$


Squaring the both parts of the obtained equality, integrating it in the domain $\Omega_{\tau}$ and using the Schwartz inequality, we receive

$$
\begin{aligned}
& \int_{\Omega_{\tau}} u_{k}^{2} d x \leq 2 \int_{\Omega_{\tau}} g_{k}^{2}(x, \gamma(x)) d x+2 \int_{\Omega_{\tau}}\left(\int_{\gamma(x)}^{\tau} \frac{\partial}{\partial t} u_{k}(x, s) d s\right)^{2} d x \\
& \leq 2 \int_{S_{\tau}} g_{k}^{2} d s+2 \int_{\Omega_{\tau}}(\tau-\gamma(x))\left[\int_{\gamma(x)}^{\tau}\left(\frac{\partial u_{k}}{\partial t}\right)^{2} d s\right] d x \leq 2 \int_{S_{\tau}} g_{k}^{2} d s \\
& \quad+2 T \int_{\Omega_{\tau}}\left[\int_{\gamma(x)}^{\tau}\left(\frac{\partial u_{k}}{\partial t}\right)^{2} d s\right] d x=2 \int_{S_{\tau}} g_{k}^{2} d s+2 T \int_{D_{\tau}}\left(\frac{\partial u_{k}}{\partial t}\right)^{2} d x d t
\end{aligned}
$$

In view of $(2.16),(2.19)$ from (2.15) it follows that

$$
\begin{aligned}
\int_{\Omega_{\tau}} & {\left[u_{k}^{2}+\left(\frac{\partial u_{k}}{\partial t}\right)^{2}+\sum_{i=1}^{n}\left(\frac{\partial u_{k}}{\partial x_{i}}\right)^{2}\right] d x \leq M_{0}\left\|g_{k}\right\|_{W_{2}^{1}\left(S_{T}\right)}^{2} } \\
& +2 \lambda M_{1}\left(\operatorname{mes}_{T}\right)^{\frac{1}{2}}\left\|g_{k}\right\|_{W_{2}^{1}\left(S_{T}\right)}+\frac{2 \lambda M_{2}}{\alpha+1} \int_{S_{T}}\left|g_{k}\right|^{\alpha+1} d s \\
& +2 \lambda M_{3} m e s \Omega_{\tau}+\left(2 \lambda M_{4}+1\right)\left[2 \int_{S_{T}} g_{k}^{2} d s+2 T \int_{D_{\tau}}\left(\frac{\partial u_{k}}{\partial t}\right)^{2} d x d t\right] \\
& +\int_{D_{\tau}}\left(\frac{\partial u_{k}}{\partial t}\right)^{2} d x d t+\int_{D_{\tau}} F_{k}^{2} d x d t \\
\leq & \left(4 \lambda M_{4} T+2 T+1\right) \int_{D_{\tau}}\left[u_{k}^{2}+\left(\frac{\partial u_{k}}{\partial t}\right)^{2}+\sum_{i=1}^{n}\left(\frac{\partial u_{k}}{\partial x_{i}}\right)^{2}\right] d x \\
& +\left[M_{0}\left\|g_{k}\right\|_{W_{2}^{1}\left(S_{T}\right)}^{2}+2 \lambda M_{1}\left(m e s S_{T}\right)^{\frac{1}{2}}\left\|g_{k}\right\|_{W_{2}^{1}\left(S_{T}\right)}+\frac{2 \lambda M_{2}}{\alpha+1} \int_{S_{T}}\left|g_{k}\right|^{\alpha+1} d s\right. \\
& \left.+2 \lambda M_{3} m e s \Omega_{\tau}+\left(4 \lambda M_{4}+2\right)\left\|g_{k}\right\|_{W_{2}^{1}\left(S_{T}\right)}^{2}+\left\|F_{k}\right\|_{L_{2}\left(D_{T}\right)}^{2}\right] .
\end{aligned}
$$

Let

$$
w(\tau):=\int_{\Omega_{\tau}}\left[u_{k}^{2}+\left(\frac{\partial u_{k}}{\partial t}\right)^{2}+\sum_{i=1}^{n}\left(\frac{\partial u_{k}}{\partial x_{i}}\right)^{2}\right] d x,
$$

then from (2.20) we have

$$
\begin{aligned}
w(\tau) \leq & \left(4 \lambda M_{4} T+2 T+1\right) \int_{0}^{\tau} w(s) d s+\left[\left(M_{0}+4 \lambda M_{4}+2\right)\left\|g_{k}\right\|_{W_{2}^{1}\left(S_{T}\right)}^{2}\right. \\
& +2 \lambda M_{1}\left(\operatorname{mes} S_{T}\right)^{\frac{1}{2}}\left\|g_{k}\right\|_{W_{2}^{1}\left(S_{T}\right)}+2 \lambda M_{3} m e s \Omega_{T} \\
& \left.+\frac{2 \lambda M_{2}}{\alpha+1} \int_{S_{T}}\left|g_{k}\right|^{\alpha+1} d s+\left\|F_{k}\right\|_{L_{2}\left(D_{T}\right)}^{2}\right], \quad 0<\tau \leq T .
\end{aligned}
$$

Hence, due to the Gronwall's lemma [28, p. 13], it follows that

$$
w(\tau) \leq A_{k} \exp \left(4 \lambda M_{4} T+2 T+1\right) \tau, \quad 0<\tau \leq T .
$$


Here

$$
\begin{aligned}
A_{k}= & \left(M_{0}+4 \lambda M_{4}+2\right) \| g_{k}||_{W_{2}^{1}\left(S_{T}\right)}^{2}+2 \lambda M_{1}\left(\text { mes } S_{T}\right)^{\frac{1}{2}}\left\|g_{k}\right\|_{W_{2}^{1}\left(S_{T}\right)} \\
& +2 \lambda M_{3} m e s \Omega_{T}+\frac{2 \lambda M_{2}}{\alpha+1} \int_{S_{T}}\left|g_{k}\right|^{\alpha+1} d s+\left\|F_{k}\right\|_{L_{2}\left(D_{T}\right)}^{2} .
\end{aligned}
$$

In view of (2.21) and (2.22) we have

$$
\left\|u_{k}\right\|_{W_{2}^{1}\left(D_{T}\right)}^{2}=\int_{0}^{T} w(\tau) d \tau \leq A_{k} T \exp \left(4 \lambda M_{4} T+2 T+1\right) T .
$$

Further, by virtue of $(2.2)-(2.5),(2.17),(2.18)$ and $(2.23)$, passing to limit for $k \rightarrow \infty$ in $(2.24)$, we obtain

$$
\|u\|_{W_{2}^{1}\left(D_{T}\right)}^{2} \leq A T \exp \left(4 \lambda M_{4} T+2 T+1\right) T \text {. }
$$

Here

$$
\begin{aligned}
A= & \left(M_{0}+4 \lambda M_{4}+2\right)\|g\|_{W_{2}^{1}\left(D_{T}\right)}^{2}+2 \lambda M_{1}\left(\text { mes } S_{T}\right)^{\frac{1}{2}}\|g\|_{W_{2}^{1}\left(S_{T}\right)} \\
& +2 \lambda M_{3} m e s \Omega_{T}+\frac{2 \lambda M_{2}}{\alpha+1} C_{1}\|g\|_{W_{2}^{1}\left(S_{T}\right)}^{2}+\|F\|_{L_{2}\left(D_{T}\right)}^{2} .
\end{aligned}
$$

Taking square root from the both sides of the inequality (2.25) and using the obvious inequality $\left(\sum_{i=1}^{m} a_{i}^{2}\right)^{\frac{1}{2}} \leq \sum_{i=1}^{m}\left|a_{i}\right|$, in view of (2.26) we finally obtain

$$
\|u\|_{W_{2}^{1}\left(D_{T}\right)} \leq c_{1}\|F\|_{L_{2}\left(D_{T}\right)}+c_{2}\|g\|_{W_{2}^{1}\left(S_{T}\right)}+c_{3}\|g\|_{W_{2}^{1}\left(S_{T}\right)}^{\frac{1}{2}}+c_{4} .
$$

Here

$$
\left\{\begin{array}{l}
c_{1}=\sqrt{T} \exp \left(2 \lambda M_{4} T+T+\frac{1}{2}\right) T \\
c_{2}=\sqrt{T}\left(M_{0}+4 \lambda M_{4}+\frac{2 \lambda M_{2}}{\alpha+1} C_{1}+2\right)^{\frac{1}{2}} \exp \left(2 \lambda M_{4} T+T+\frac{1}{2}\right) T \\
c_{3}=\sqrt{T} \sqrt{2 \lambda M_{1}}\left(\operatorname{mes}_{T}\right)^{\frac{1}{4}} \exp \left(2 \lambda M_{4} T+T+\frac{1}{2}\right) T \\
c_{4}=\sqrt{T}\left(2 \lambda M_{3} m e s \Omega_{T}\right)^{\frac{1}{2}} \exp \left(2 \lambda M_{4} T+T+\frac{1}{2}\right) T
\end{array}\right.
$$

This completely proves Lemma 2.1 .

\section{Global solvability of the problem (1.1), (1.2) of the class $W_{2}^{1}$}

First consider a solvability of a linear problem corresponding to the problem (1.1), (1.2) when $\lambda=0$ in the equation (1.1), i.e. for the problem

$$
\begin{aligned}
L_{0} u(x, t) & =F(x, t),(x, t) \in D_{T}, \\
u(x, t) & =g(x, t),(x, t) \in S_{T} .
\end{aligned}
$$

In this case for $F \in L_{2}\left(D_{T}\right), g \in W_{2}^{1}\left(S_{T}\right)$ we introduce analogously a notion of strong generalized solution $u \in W_{2}^{1}\left(D_{T}\right)$ of the problem (3.1), (3.2) of the class $W_{2}^{1}$ in the domain $D_{T}$, for which there exists a sequence of functions $u_{k} \in C^{2}\left(\bar{D}_{T}\right)$, such that $u_{k} \rightarrow u$ in the space $W_{2}^{1}\left(D_{T}\right), L_{0} u_{k} \rightarrow F$ in the space $L_{2}\left(D_{T}\right)$ and $\left.u_{k}\right|_{S_{T}} \rightarrow g$ in the space $W_{2}^{1}\left(S_{T}\right)$. Note that, as it follows from Lemma 2.1, due to (2.27) when $\lambda=0$ for the strong generalized solution $u \in W_{2}^{1}\left(D_{T}\right)$ of the problem (3.1), (3.2) the following estimate

$$
\|u\|_{W_{2}^{1}\left(D_{T}\right)} \leq c\left(\|F\|_{L_{2}\left(D_{T}\right)}+\|g\|_{W_{2}^{1}\left(S_{T}\right)}\right)
$$


is valid, where

$$
c=\sqrt{T}\left(M_{0}+2\right)^{\frac{1}{2}} \exp \left(T+\frac{1}{2}\right) T .
$$

Let us introduce into consideration the weighted Sobolev space $W_{2, \alpha}^{k}(D)$, $0<\alpha<\infty, k=1,2, \ldots$, consisting of the functions from the class $W_{2, l o c}^{k}(D)$ for which the norm [29]

$$
\|u\|_{W_{2, \alpha}^{k}(D)}^{2}=\sum_{i=0}^{k} \int_{D} r^{-2 \alpha-2(k-i)}\left|\frac{\partial^{i} u}{\partial x^{\hat{i}} \partial t^{i_{0}}}\right|^{2} d x d t,
$$

is finite, where

$$
r=\left(\sum_{j=1}^{n} x_{j}^{2}+t^{2}\right)^{\frac{1}{2}}, \quad \frac{\partial^{i} u}{\partial x^{\hat{i}} \partial t^{i_{0}}}=\frac{\partial^{i} u}{\partial x^{i_{1}} \ldots \partial x^{i_{n}} \partial t^{i_{0}}}, \quad i=i_{1}+\cdots+i_{n}+i_{0} .
$$

Analogously is introduced the space $W_{2, \alpha}^{k}(S), S=\partial D$ [29].

Together with the problem (3.1), (3.2) consider an analogous problem in the infinite cone $D$ with the following statement

$$
\begin{aligned}
L_{0} u(x, t) & =F(x, t),(x, t) \in D, \\
u(x, t) & =g(x, t),(x, t) \in S .
\end{aligned}
$$

According to (1.3) and the result of work [30, p. 114] there exists constant $\alpha_{0}=\alpha_{0}(k)>1$, such that for $\alpha \geq \alpha_{0}$ the problem (3.5), (3.6) has the unique solution $u \in W_{2, \alpha}^{k}(D)$ for every $F \in W_{2, \alpha-1}^{k-1}(D)$ and $g \in W_{2, \alpha-\frac{1}{2}}^{k}(S), k \geq 2$.

Since the space of finite and infinitely differentiable functions $C_{0}^{\infty}\left(D_{T}\right)$ is dense in $L_{2}\left(D_{T}\right)$, then for a given $F \in L_{2}\left(D_{T}\right)$ there exists a sequence of functions $F_{l} \in C_{0}^{\infty}\left(D_{T}\right)$, such that

$$
\lim _{l \rightarrow \infty}\left\|F_{l}-F\right\|_{L_{2}\left(D_{T}\right)}=0 .
$$

For fixed $l$, extending the function $F_{l}$ with zero beyond domain $D_{T}$ and using the same notation for it, we will have $F_{l} \in C_{0}^{\infty}(D)$. It is obvious that $F_{l} \in W_{2, \alpha-1}^{k-1}(D)$ for every $k \geq 2$ and $\alpha>1$, and, therefore, for $\alpha \geq \alpha_{0}=\alpha_{0}(k)$ too. If $g \in W_{2}^{1}\left(S_{T}\right)$, then there exists a function $\tilde{g} \in W_{2}^{1}(S)$ such that $g=\left.\tilde{g}\right|_{S_{T}}$ and diam supp $\tilde{g}<\infty[25$, p. 56]. At the same time the space $C_{*}^{\infty}(S):=\left\{g \in C^{\infty}(S)\right.$ : diam supp $g<+\infty, 0 \neq$ supp $\left.g\right\}$ is dense in $W_{2}^{1}(S)$ [31]. Therefore there exists a sequence of functions $g_{l} \in C_{*}^{\infty}(S)$ such that $\lim _{l \rightarrow \infty}\left\|g_{l}-\tilde{g}\right\|_{W_{2}^{1}(S)}=0$. It is easy to see that $g_{l} \in W_{2, \alpha-\frac{1}{2}}^{k}(S)$ for every $k \geq 2$ and $\alpha>1$, and therefore for $\alpha \geq \alpha_{0}=\alpha_{0}(k)$ too. In view of the aforesaid there exists the solution $\tilde{u}_{l} \in W_{2, \alpha}^{k}(D)$ of the problem (3.5), (3.6) for $F=F_{l}$ and $g=g_{l}$. Let $u_{l}=\left.\tilde{u}_{l}\right|_{D_{T}}$. Since $u_{l} \in W_{2}^{k}\left(D_{T}\right)$, then choosing number $k$ sufficiently large, namely $k>\frac{n+1}{2}+2$, according to the theorem of embedding $\left[25\right.$, p. 84] we have $u_{l} \in C^{2}\left(\bar{D}_{T}\right)$. According to the estimate (3.3) we have

$$
\left\|u_{l}-u_{l^{\prime}}\right\|_{W_{2}^{1}\left(D_{T}\right)} \leq c\left(\left\|F_{l}-F_{l^{\prime}}\right\|_{L_{2}\left(D_{T}\right)}+\left\|g_{l}-g_{l^{\prime}}\right\|_{W_{2}^{1}\left(S_{T}\right)}\right) .
$$


Since the sequences $\left\{F_{l}\right\}$ and $\left\{g_{l}\right\}$ are fundamental in the spaces $L_{2}\left(D_{T}\right)$ and $W_{2}^{1}\left(S_{T}\right)$, respectively, then due to (3.7) the sequence $\left\{u_{l}\right\}$ is fundamental in the space $W_{2}^{1}\left(D_{T}\right)$. Therefore, because of completeness of the space $W_{2}^{1}\left(D_{T}\right)$ there exists the function $u \in W_{2}^{1}\left(D_{T}\right)$ such that $\lim _{l \rightarrow \infty}\left\|u_{l}-u\right\|_{W_{2}^{1}\left(D_{T}\right)}=0$, and since $L_{0} u_{l}=F_{l} \rightarrow F$ in the space $W_{2}^{1}\left(D_{T}\right)$ and $g_{l}=\left.u_{l}\right|_{S_{T}} \rightarrow g$ in the space $W_{2}^{1}\left(S_{T}\right)$, then the function $u$ will be a strong generalized solution of the problem (3.1), (3.2) of the class $W_{2}^{1}$ in the domain $D_{T}$. The uniqueness of this solution of the problem (3.1), (3.2) of the class $W_{2}^{1}$ in the domain $D_{T}$ follows from a priori estimate (3.3). Therefore, for the solution $u$ of the problem (3.1), (3.2) we have $u=L_{0}^{-1}(F, g)$, where $L_{0}^{-1}: L_{2}\left(D_{T}\right) \times W_{2}^{1}\left(S_{T}\right) \rightarrow W_{2}^{1}\left(D_{T}\right)$ is a linear continuous operator, whose norm, in view of (3.3), admits the following estimate

$$
\left\|L_{0}^{-1}\right\|_{L_{2}\left(D_{T}\right) \times W_{2}^{1}\left(S_{T}\right) \rightarrow W_{2}^{1}\left(D_{T}\right)} \leq c,
$$

where constant $c$ is determined from (3.4).

Because of linearity of the operator $L_{0}^{-1}: L_{2}\left(D_{T}\right) \times W_{2}^{1}\left(S_{T}\right) \rightarrow W_{2}^{1}\left(D_{T}\right)$ we have the following representation

$$
L_{0}^{-1}(F, g)=L_{01}^{-1}(F)+L_{02}^{-1}(g),
$$

where $L_{01}^{-1}: L_{2}\left(D_{T}\right) \rightarrow W_{2}^{1}\left(D_{T}\right)$ and $L_{02}^{-1}: W_{2}^{1}\left(S_{T}\right) \rightarrow W_{2}^{1}\left(D_{T}\right)$ are the linear continuous operators, which satisfy conditions

$$
\left\|L_{01}^{-1}\right\|_{L_{2}\left(D_{T}\right) \rightarrow W_{2}^{1}\left(D_{T}\right)} \leq c, \quad\left\|L_{02}^{-1}\right\|_{W_{2}^{1}\left(S_{T}\right) \rightarrow W_{2}^{1}\left(D_{T}\right)} \leq c
$$

on the basis of (3.8).

Remark 3.1. Note that for $F \in L_{2}\left(D_{T}\right), g \in W_{2}^{1}\left(S_{T}\right), 0<\alpha<\frac{n+1}{n-1}$, by virtue of (3.8), (3.9), (3.10) and Remark 1.2, the function $u \in W_{2}^{1}\left(D_{T}\right)$ is a strong generalized solution of the problem (1.1), (1.2) of the class $W_{2}^{1}$ in the domain $D_{T}$ if and only if $u$ satisfies the following functional equation

$$
u=L_{01}^{-1}(-\lambda f(u))+L_{01}^{-1}(F)+L_{02}^{-1}(g)
$$

in the space $W_{2}^{1}\left(D_{T}\right)$.

Rewrite (3.11) in the form

$$
u=A_{0} u:=-L_{01}^{-1}\left(K_{0} u\right)+L_{01}^{-1}(F)+L_{02}^{-1}(g),
$$

where the operator $K_{0}: W_{2}^{1}\left(D_{T}\right) \rightarrow L_{2}\left(D_{T}\right)$ from (1.6), according to Remark 1.2, is a continuous and compact operator. Therefore, in view of (3.10) the operator $A_{0}: W_{2}^{1}\left(D_{T}\right) \rightarrow W_{2}^{1}\left(D_{T}\right)$ is also continuous and compact. At the same time, because of Lemma 2.1 and (2.11), (2.27), for every parameter $\tau \in[0,1]$ and every solution $u$ of the equation $u=\tau A_{0} u$ with parameter $\tau$ it is valid the same a priori estimate (2.1) with nonnegative constants $c_{i}$, not depending on $u, F, g$ and $\tau$. Thus, according to the Leray-Schauder theorem [32, p. 375], the equation (3.12), and therefore, according to Remark 3.1 the problem (1.1), (1.2) has at least one solution $u \in W_{2}^{1}\left(D_{T}\right)$. We proved the following theorem. 
Theorem 3.2. Let $\lambda>0$ and the conditions (1.3), (1.4), (1.5) with $0<\alpha<\frac{n+1}{n-1}$ be fulfilled. Then for every $F \in L_{2}\left(D_{T}\right)$ and $g \in W_{2}^{1}\left(S_{T}\right)$ the problem (1.1), (1.2) has at least one strong generalized solution $u$ of the class $W_{2}^{1}$ in the domain $D_{T}$.

From Theorem 3.2 it follows the global solvability of the problem (1.1), (1.2) of the class $W_{2}^{1}$ in the sense of Definition 1.4.

\section{Uniqueness of the solution of the problem (1.1), (1.2) of the class $W_{2}^{1}$}

Let us impose on the function $f$ in the equation (1.1) additional requirement

$$
f \in C^{1}(\mathbb{R}), \quad\left|f^{\prime}(u)\right| \leq a+b|u|^{\gamma} ; \quad a, b, \gamma=\text { const } \geq 0 .
$$

It is obvious that the condition (1.4) with $\alpha=\gamma+1$ follows from (4.1), and when $\gamma<\frac{2}{n-1}$ we have $\alpha=\gamma+1<\frac{n+1}{n-1}$.

Theorem 4.1. Let $0 \leq \gamma<\frac{2}{n-1}$ and the conditions (1.3), (4.1) be fulfilled. Then the problem (1.1), (1.2) cannot have more than one strong generalized solution of the class $W_{2}^{1}$ in the domain $D_{T}$ in the sense of Definition 1.3.

Proof. Suppose that $F \in L_{2}\left(D_{T}\right), g \in W_{2}^{1}\left(S_{T}\right)$ and the problem (1.1), (1.2) has two strong generalized solutions $u_{1}, u_{2} \in W_{2}^{1}\left(D_{T}\right)$ in the sense of Definition 1.3, i.e. there exist two sequences of the functions $u_{i k} \in C^{2}\left(\bar{D}_{T}\right), i=$ 1,$2 ; k=1,2, \ldots$ such that

$$
\begin{aligned}
& \lim _{k \rightarrow \infty}\left\|u_{i k}-u_{i}\right\|_{W_{2}^{1}\left(D_{T}\right)}=0, \quad \lim _{k \rightarrow \infty}\left\|L_{\lambda} u_{i k}-F\right\|_{L_{2}\left(D_{T}\right)}=0, i=1,2, \\
& \lim _{k \rightarrow \infty}\left\|g_{i k}-g\right\|_{W_{2}^{1}\left(S_{T}\right)}=0, g_{i k}=\left.u_{i k}\right|_{S_{T}}, i=1,2 .
\end{aligned}
$$

Let

$$
w=u_{2}-u_{1}, \quad w_{k}=u_{2 k}-u_{1 k} \quad F_{k}=L_{\lambda} u_{2 k}-L_{\lambda} u_{1 k} \quad g_{k}^{0}=\left.w_{k}\right|_{S_{T}} .
$$

Then in view of (4.2), (4.3) and (4.4) we have

$$
\begin{gathered}
\lim _{k \rightarrow \infty}\left\|w_{k}-w\right\|_{W_{2}^{1}\left(D_{T}\right)}=0, \quad \lim _{k \rightarrow \infty}\left\|F_{k}\right\|_{L_{2}\left(D_{T}\right)}=0, \\
\lim _{k \rightarrow \infty}\left\|g_{k}^{0}\right\|_{W_{2}^{1}\left(S_{T}\right)}=0 .
\end{gathered}
$$

In accordance with (4.4) we consider the function $w_{k} \in C^{2}\left(\bar{D}_{T}\right)$ as a solution of the following problem

$$
\begin{aligned}
\square w_{k} & :=\frac{\partial^{2} w_{k}}{\partial t^{2}}-\triangle w_{k}=-\lambda\left[f\left(u_{2 k}\right)-f\left(u_{1 k}\right)\right]+F_{k}, \\
\left.w_{k}\right|_{S_{T}} & =g_{k}^{0} .
\end{aligned}
$$


Multiplying the both sides of the equation (4.7) by the function $\frac{\partial w_{k}}{\partial t}$, after integration in the domain $D_{\tau}$, in the same way as in derivation of the equality (2.9) we have

$$
\begin{aligned}
& \int_{D_{\tau}}\left\{F_{k}-\lambda\left[f\left(u_{2 k}\right)-f\left(u_{1 k}\right)\right]\right\} \frac{\partial w_{k}}{\partial t} d x d t \\
& =\int_{S_{\tau}} \frac{1}{2 \nu_{0}}\left[\sum_{i=1}^{n}\left(\frac{\partial w_{k}}{\partial x_{i}} \nu_{0}-\frac{\partial w_{k}}{\partial t} \nu_{i}\right)^{2}+\left(\frac{\partial w_{k}}{\partial t}\right)^{2}\left(\nu_{0}^{2}-\sum_{j=1}^{n} \nu_{j}^{2}\right)\right] d s \\
& \quad+\frac{1}{2} \int_{\Omega_{\tau}}\left[\left(\frac{\partial w_{k}}{\partial t}\right)^{2}+\sum_{i=1}^{n}\left(\frac{\partial w_{k}}{\partial x_{i}}\right)^{2}\right] d x, 0<\tau \leq T .
\end{aligned}
$$

Due to (1.3) and (2.12), (2.13) from (4.9), taking into account (4.8), we obtain

$$
\begin{aligned}
\int_{\Omega_{\tau}} & {\left[\left(\frac{\partial w_{k}}{\partial t}\right)^{2}+\sum_{i=1}^{n}\left(\frac{\partial w_{k}}{\partial x_{i}}\right)^{2}\right] d x \leq M_{0}\left\|g_{k}^{0}\right\|_{W_{2}^{1}\left(S_{T}\right)}^{2} } \\
& +2 \int_{D_{\tau}} F_{k} \frac{\partial w_{k}}{\partial t} d x d t+2|\lambda| \int_{D_{\tau}}\left|f\left(u_{2 k}\right)-f\left(u_{1 k}\right)\right|\left|\frac{\partial w_{k}}{\partial t}\right| d x d t .
\end{aligned}
$$

Taking into account the inequality $\left|d_{1}+d_{2}\right|^{\gamma} \leq 2^{\gamma} \max \left(\left|d_{1}\right|^{\gamma},\left|d_{2}\right|^{\gamma}\right) \leq$ $2^{\gamma}\left(\left|d_{1}\right|^{\gamma}+\left|d_{2}\right|^{\gamma}\right)$ for $\gamma \geq 0$, due to (4.1) we have

$$
\begin{aligned}
& \left|f\left(u_{2 k}\right)-f\left(u_{1 k}\right)\right|=\left|\left(u_{2 k}-u_{1 k}\right) \int_{0}^{1} f^{\prime}\left(u_{1 k}+t\left(u_{2 k}-u_{1 k}\right)\right) d t\right| \\
& \leq\left|u_{2 k}-u_{1 k}\right| \int_{0}^{1}\left(a+b\left|(1-t) u_{1 k}+t u_{2 k}\right|^{\gamma}\right) d t \leq a\left|u_{2 k}-u_{1 k}\right| \\
& \quad+2^{\gamma} b\left|u_{2 k}-u_{1 k}\right|\left(\left|u_{1 k}\right|^{\gamma}+\left|u_{2 k}\right|^{\gamma}\right)=a\left|w_{k}\right|+2^{\gamma} b\left|w_{k}\right|\left(\left|u_{1 k}\right|^{\gamma}+\left|u_{2 k}\right|^{\gamma}\right) .
\end{aligned}
$$

Applying the obvious inequality $2 d_{1} d_{2} \leq d_{1}^{2}+d_{2}^{2}$ and the inequality (4.11) to the right-side of (4.10) we obtain

$$
\begin{aligned}
\int_{\Omega_{\tau}} & {\left[\left(\frac{\partial w_{k}}{\partial t}\right)^{2}+\sum_{i=1}^{n}\left(\frac{\partial w_{k}}{\partial x_{i}}\right)^{2}\right] d x \leq M_{0}|| g_{k}^{0} \|_{W_{2}^{1}\left(S_{T}\right)}^{2}+\int_{D_{T}} F_{k}^{2} d x d t } \\
& +\int_{D_{T}}\left(\frac{\partial w_{k}}{\partial t}\right)^{2} d x d t+a|\lambda| \int_{D_{T}}\left[w_{k}^{2}+\left(\frac{\partial w_{k}}{\partial t}\right)^{2}\right] d x d t \\
& +2^{\gamma+1} b|\lambda| \int_{D_{T}}\left(\left|u_{1 k}\right|^{\gamma}+\left|u_{2 k}\right|^{\gamma}\right)\left|w_{k}\right|\left|\frac{\partial w_{k}}{\partial t}\right| d x d t .
\end{aligned}
$$

The last integral in the right-side of (4.12) can be estimated by the Holder inequality

$$
\begin{gathered}
\int_{D_{T}}\left(\left|u_{1 k}\right|^{\gamma}+\left|u_{2 k}\right|^{\gamma}\right)\left|w_{k}\right|\left|\frac{\partial w_{k}}{\partial t}\right| d x d t \leq\left(\left\|\left|u_{1 k}\right|^{\gamma}\right\|_{L_{n+1}\left(D_{T}\right)}\right. \\
\left.+\left\|\left|u_{2 k}\right|^{\gamma}\right\|_{L_{n+1}\left(D_{T}\right)}\right)|| w_{k}\left\|_{L_{p}\left(D_{T}\right)}|| \frac{\partial w_{k}}{\partial t}\right\|_{L_{2}\left(D_{T}\right)}
\end{gathered}
$$


where $\frac{1}{n+1}+\frac{1}{p}+\frac{1}{2}=1$, i.e. for

$$
p=\frac{2(n+1)}{n-1} .
$$

Since $\operatorname{dim} D_{T}=n+1$, then according to the embedding theorem of Sobolev [27, p. 111] for $1 \leq q \leq \frac{2(n+1)}{n-1}$ we have

$$
\|v\|_{L_{q}\left(D_{T}\right)} \leq C_{q}\|v\|_{W_{2}^{1}\left(D_{T}\right)} \forall v \in W_{2}^{1}\left(D_{T}\right)
$$

with positive constant $C_{q}$, not depending on $v \in W_{2}^{1}\left(D_{T}\right)$.

According to the conditions of the theorem $\gamma<\frac{2}{n-1}$ and, therefore, $\gamma(n+1)<\frac{2(n+1)}{n-1}$. Thus, in view of (4.14) and inequality (4.15) we have

$$
\begin{aligned}
& \left\|\left|u_{i k}\right|^{\gamma}\right\|_{L_{n+1}\left(D_{T}\right)}=\left\|u_{i k}\right\|_{L_{\gamma(n+1)}\left(D_{T}\right)} \\
& \quad \leq C_{\gamma(n+1)}^{\gamma}\left\|u_{i k}\right\|_{W_{2}^{1}\left(D_{T}\right)}^{\gamma}, \quad i=1,2 ; k \geq 1, \\
& \left\|w_{k}\right\|_{L_{p}\left(D_{T}\right)} \leq C_{p}\left\|w_{k}\right\|_{W_{2}^{1}\left(D_{T}\right)}, \quad p=\frac{2(n+1)}{n-1}, k \geq 1 .
\end{aligned}
$$

According to the first equality of (4.2) there exists the natural number $k_{0}$ such that for $k \geq k_{0}$ we will have

$$
\left\|u_{i k}\right\|_{W_{2}^{1}\left(D_{T}\right)}^{\gamma} \leq\left\|u_{i}\right\|_{W_{2}^{1}\left(D_{T}\right)}^{\gamma}+1, \quad i=1,2 ; k \geq k_{0} .
$$

Whence, taking into account (4.13), (4.16) and (4.17), it follows that

$$
\begin{aligned}
& 2^{\gamma+1} b|\lambda| \int_{D_{T}}\left(\left|u_{1 k}\right|^{\gamma}+\left|u_{2 k}\right|^{\gamma}\right)\left|w_{k}\right|\left|\frac{\partial w_{k}}{\partial t}\right| d x d t \\
& \leq 2^{\gamma+1} b|\lambda| C_{\gamma(n+1)}^{\gamma}\left(\left\|u_{1}\right\|_{W_{2}^{1}\left(D_{T}\right)}^{\gamma}\right. \\
& \left.\quad+\left\|u_{2}\right\|_{W_{2}^{1}\left(D_{T}\right)}^{\gamma}+2\right) C_{p}\left\|w_{k}\right\|_{W_{2}^{1}\left(D_{T}\right)}|| \frac{\partial w_{k}}{\partial t} \|\left.\right|_{L_{2}\left(D_{T}\right)} \\
& \leq M_{1}^{\star}\left(\left\|w_{k}\right\|_{W_{2}^{1}\left(D_{T}\right)}^{2}+\left\|\frac{\partial w_{k}}{\partial t}\right\|_{L_{2}\left(D_{T}\right)}^{2}\right) \leq 2 M_{1}^{\star}\left\|w_{k}\right\|_{W_{2}^{1}\left(D_{T}\right)}^{2} \\
& =2 M_{1}^{\star} \int_{D_{T}}\left[w_{k}^{2}+\left(\frac{\partial w_{k}}{\partial t}\right)^{2}+\sum_{i=1}^{n}\left(\frac{\partial w_{k}}{\partial x_{i}}\right)^{2}\right] d x d t,
\end{aligned}
$$

where $2 M_{1}^{\star}=2^{\gamma+1} b|\lambda| C_{\gamma(n+1)}^{\gamma}\left(\left\|u_{1}\right\|_{W_{2}^{1}\left(D_{T}\right)}^{\gamma}+\left\|u_{2}\right\|_{W_{2}^{1}\left(D_{T}\right)}^{\gamma}+2\right) C_{p}$.

In view of (4.18) from (4.12) we have

$$
\begin{aligned}
& \int_{\Omega_{\tau}}\left[\left(\frac{\partial w_{k}}{\partial t}\right)^{2}+\sum_{i=1}^{n}\left(\frac{\partial w_{k}}{\partial x_{i}}\right)^{2}\right] d x \leq M_{2}^{\star} \\
& \quad \times \int_{D_{T}}\left[w_{k}^{2}+\left(\frac{\partial w_{k}}{\partial t}\right)^{2}+\sum_{i=1}^{n}\left(\frac{\partial w_{k}}{\partial x_{i}}\right)^{2}\right] d x d t \\
& +M_{0}\left\|g_{k}^{0}\right\|_{W_{2}^{1}\left(S_{T}\right)}^{2}+\int_{D_{T}} F_{k}^{2} d x d t, \quad 0<\tau \leq T,
\end{aligned}
$$

where $M_{2}^{\star}=1+a|\lambda|+2 M_{1}^{\star}$. 
Note that the inequality (2.19) is still valid if we replace $u_{k}$ and $g_{k}$ with $w_{k}$ and $g_{k}^{0}$, i.e.

$$
\begin{aligned}
& \int_{\Omega_{\tau}} w_{k}^{2} d x \leq 2 \int_{S_{T}}\left(g_{k}^{0}\right)^{2} d s+2 T \int_{D_{T}}\left(\frac{\partial w_{k}}{\partial t}\right)^{2} d x d t \\
& \leq 2\left\|g_{k}^{0}\right\|_{W_{2}^{1}\left(S_{T}\right)}^{2}+2 T \int_{D_{T}}\left[w_{k}^{2}+\left(\frac{\partial w_{k}}{\partial t}\right)^{2}+\sum_{i=1}^{n}\left(\frac{\partial w_{k}}{\partial x_{i}}\right)^{2}\right] d x .
\end{aligned}
$$

Putting

$$
v_{k}(\tau):=\int_{\Omega_{\tau}}\left[w_{k}^{2}+\left(\frac{\partial w_{k}}{\partial t}\right)^{2}+\sum_{i=1}^{n}\left(\frac{\partial w_{k}}{\partial x_{i}}\right)^{2}\right] d x
$$

and summing up the inequalities (4.19) and (4.20), we receive

$$
v_{k}(\tau) \leq\left(M_{2}^{\star}+2 T\right) \int_{0}^{\tau} v(s) d s+\left(M_{0}+2\right)\left\|g_{k}^{0}\right\|_{W_{2}^{1}\left(S_{T}\right)}^{2}+\left\|F_{k}\right\|_{L_{2}\left(D_{T}\right)}^{2} .
$$

Using the Gronwall's lemma [28, p. 13] we conclude that

$$
v_{k}(\tau) \leq\left[\left(M_{0}+2\right)\left\|g_{k}^{0}\right\|_{W_{2}^{1}\left(S_{T}\right)}^{2}+\left\|F_{k}\right\|_{L_{2}\left(D_{T}\right)}^{2}\right] \exp \left(M_{2}^{\star}+2 T\right) \tau .
$$

From (4.21) and (4.22) it follows that

$$
\begin{aligned}
\left\|w_{k}\right\|_{W_{2}^{1}\left(D_{T}\right)}^{2}= & \int_{0}^{T} v_{k}(\tau) d \tau \leq T\left[\left(M_{0}+2\right)\left\|g_{k}^{0}\right\|_{W_{2}^{1}\left(S_{T}\right)}^{2}\right. \\
& \left.+\left\|F_{k}\right\|_{L_{2}\left(D_{T}\right)}^{2}\right] \exp \left(M_{2}^{\star}+2 T\right) T
\end{aligned}
$$

Due to (4.5) and (4.6) from (4.23) we have

$$
\lim _{k \rightarrow \infty}\left\|w_{k}\right\|_{W_{2}^{1}\left(D_{T}\right)}=0 .
$$

Hence, in view of the first equality of (4.5), it follows that

$$
\begin{aligned}
\|w\|_{W_{2}^{1}\left(D_{T}\right)} & =\lim _{k \rightarrow \infty}\left\|w-w_{k}+w_{k}\right\|_{W_{2}^{1}\left(D_{T}\right)} \\
& \leq \lim _{k \rightarrow \infty}\left\|w-w_{k}\right\|_{W_{2}^{1}\left(D_{T}\right)}+\lim _{k \rightarrow \infty}\left\|w_{k}\right\|_{W_{2}^{1}\left(D_{T}\right)}=0 .
\end{aligned}
$$

Therefore $w=u_{2}-u_{1}=0$, i.e. $u_{2}=u_{1}$, which proves Theorem 4.1.

The theorem below immediately follows from Theorems 3.2 and 4.1.

Theorem 4.2. Let $\lambda>0$ and the conditions (1.3), (4.1), (1.5) with $0 \leq \gamma<\frac{2}{n-1}$ be fulfilled. Then for every $F \in L_{2}\left(D_{T}\right)$ and $g \in W_{2}^{1}\left(S_{T}\right)$ the problem (1.1), (1.2) has the unique strong generalized solution $u$ of the class $W_{2}^{1}$ in the domain $D_{T}$. 


\section{Absence of the global solvability of the problem (1.1), (1.2)}

Below we show that violation of the condition (1.5) may cause an absence of the global solvability of the problem (1.1), (1.2).

Lemma 5.1. Let $u$ be a strong generalized solution of the problem (1.1), (1.2) of the class $W_{2}^{1}$ in the domain $D_{T}$ in the sense of Definition 1.3. Then the following integral equality

$$
\int_{D_{T}} u \square \varphi d x d t=-\lambda \int_{D_{T}} f(u) \varphi d x d t+\int_{D_{T}} F \varphi d x d t
$$

is valid for every function $\varphi$ satisfying conditions

$$
\varphi \in C^{2}\left(\bar{D}_{T}\right),\left.\quad \varphi\right|_{\partial D_{T}}=\left.\frac{\partial \varphi}{\partial \nu}\right|_{\partial D_{T}}=0
$$

where $\square:=\frac{\partial^{2}}{\partial t^{2}}-\triangle, \nu$ is the unit vector of the outer normal to $\partial D_{T}$.

Proof. According to the definition of a strong generalized solution $u$ of the problem (1.1), (1.2) of the class $W_{2}^{1}$ in the domain $D_{T}$ there exists the sequence $u_{k} \in C^{2}\left(\bar{D}_{T}\right)$ such that the equalities (2.2)-(2.6) are valid. Let us multiply the both part of the equality (2.4) by the function $\varphi$ and integrate the received equality in the domain $D_{T}$. Due to (5.2) integration by parts of the left-side of this equality yields

$$
\int_{D_{T}} u_{k} \square \varphi d x d t+\lambda \int_{D_{T}} f\left(u_{k}\right) \varphi d x d t=\int_{D_{T}} F \varphi d x d t .
$$

Passing in the equality (5.3) to limit for $k \rightarrow \infty$ and taking into account (2.2) and Definition 1.3 we obtain the equality (5.1).

Let the nonlinear function $f$ together with (1.4) satisfy the condition

$$
f(u) \geq m_{0}|u|^{\alpha}, \quad m_{0}=\text { const }>0, \alpha>1 .
$$

It is obvious that in this case the condition (1.5) will be violated since $\int_{0}^{u} f(s) d s$ $\leq \frac{m_{0}}{\alpha+1}|u|^{\alpha+1}$ signu for $u<0$, and because $\alpha>1$, then for sufficiently large negative $u$ the inequality (1.5) is not valid.

Let us use the method of test functions [14, pp. 10-12].

Let us introduce into consideration the function $\varphi^{0}=\varphi^{0}(x, t)$ such that

$$
\varphi^{0} \in C^{2}\left(\bar{D}_{\infty}\right),\left.\varphi^{0}\right|_{D_{T=1}}>0,\left.\varphi^{0}\right|_{t \geq 1}=0,\left.\varphi^{0}\right|_{\partial D_{T=1}}=\left.\frac{\partial \varphi^{0}}{\partial \nu}\right|_{\partial D_{T=1}}=0
$$

and

$$
æ_{0}:=\int_{D_{T=1}} \frac{\left|\square \varphi^{0}\right|^{\alpha^{\prime}}}{\left|\varphi^{0}\right|^{\alpha^{\prime}-1}} d x d t<+\infty, \frac{1}{\alpha}+\frac{1}{\alpha^{\prime}}=1 .
$$

It is easy to verify that for sufficiently large positive $m$ and $k$ for the function $\varphi^{0}$, satisfying conditions (5.5) and (5.6), may be chosen the function

$$
\varphi^{0}(x, t)=\left\{\begin{array}{l}
\omega^{m}\left(\frac{x}{t}\right)(1-t)^{m} t^{k}, \quad(x, t) \in D_{T=1}, \\
0, \quad t \geq 1,
\end{array}\right.
$$

where the function $\omega \in C^{\infty}\left(\mathbb{R}^{n}\right)$ defines an equation of the conic section $\partial \Omega_{1}=$ $S \cap\{t=1\}: \omega(x)=0,\left.\nabla \omega\right|_{\partial \Omega_{1}} \neq 0$, besides $\left.\omega\right|_{\Omega_{1}}>0, \Omega_{1}: D \cap\{t=1\}$. 
Setting that $\varphi_{T}(x, t):=\varphi^{0}\left(\frac{x}{T}, \frac{t}{T}\right), T>0$, due to $(5.5)$ it is easy to see that

$$
\varphi_{T} \in C^{2}\left(\bar{D}_{T}\right),\left.\quad \varphi_{T}\right|_{D_{T}}>0,\left.\quad \varphi_{T}\right|_{\partial D_{T}}=\left.\frac{\partial \varphi_{T}}{\partial \nu}\right|_{\partial D_{T}}=0 .
$$

Putting that the function $F$ is fixed, let us introduce into consideration the function of one variable $\mathrm{T}$

$$
\zeta(T):=-\int_{D_{T}} F \varphi_{T} d x d t, \quad T>0 .
$$

Theorem 5.2. Let $\lambda>0,1<\alpha<\frac{n+1}{n-1}$, the conditions (1.4), (5.4) be fulfilled, $F \in L_{2, l o c}(D), F \leq 0$ and $\left.F\right|_{D_{T}} \in L_{2}\left(D_{T}\right)$ for every $T>0$, and $g=0$. Then if

$$
\lim _{T \rightarrow+\infty} \inf \zeta(T)>0,
$$

then there exists the positive number $T_{0}=T_{0}(F)$ such that for $T>T_{0}$ the problem (1.1), (1.2) cannot have a strong generalized solution of the class $W_{2}^{1}$ in the domain $D_{T}$.

Proof. In view of (5.7), assuming in the equality (5.1) $\varphi=\varphi_{T}$ and taking into account $\lambda>0,(5.4)$ and (5.8), we receive

$$
\begin{aligned}
& \lambda_{1} \int_{D}|u|^{\alpha} \varphi_{T} d x d t \leq-\int_{D_{T}} u \square \varphi_{T} d x d t-\zeta(T) \\
& \quad \leq \int_{D_{T}}|u|\left|\square \varphi_{T}\right| d x d t-\zeta(T), \quad \lambda_{1}=\lambda m_{0}>0 .
\end{aligned}
$$

If in Young's inequality with the parameter $\varepsilon>0$

$$
a b \leq \frac{\varepsilon}{\alpha} a^{\alpha}+\frac{1}{\alpha^{\prime} \varepsilon^{\alpha^{\prime}-1}} b^{\alpha^{\prime}} ; \quad a, b \geq 0, \frac{1}{\alpha}+\frac{1}{\alpha^{\prime}}=1,
$$

we take $a=|u| \varphi_{T}^{\frac{1}{\alpha}}, b=\frac{\left|\square \varphi_{T}\right|}{\varphi_{T}^{\frac{1}{\alpha}}}$, then taking into account equality $\frac{\alpha \prime}{\alpha}=\alpha-1$, we have

$$
|u|\left|\square \varphi_{T}\right|=|u| \varphi_{T}^{\frac{1}{\alpha}} \frac{\left|\square \varphi_{T}\right|}{\varphi_{T}^{\frac{1}{\alpha}}} \leq \frac{\varepsilon}{\alpha}|u|^{\alpha} \varphi_{T}+\frac{1}{\alpha^{\prime} \varepsilon^{\alpha^{\prime}-1}} \frac{\left|\square \varphi_{T}\right|^{\alpha^{\prime}}}{\varphi_{T}^{\alpha^{\prime}-1}} .
$$

In view of (5.10) and (5.11) we have

$$
\left(\lambda_{1}-\frac{\varepsilon}{\alpha}\right) \int_{D_{T}}|u|^{\alpha} \varphi_{T} d x d t \leq \frac{1}{\alpha^{\prime} \varepsilon^{\alpha^{\prime}-1}} \int_{D_{T}} \frac{\left|\square \varphi_{T}\right|^{\alpha^{\prime}}}{\varphi_{T}^{\alpha^{\prime}-1}} d x d t-\zeta(T),
$$

whence for $\varepsilon<\left|\lambda_{1}\right| \alpha$ we obtain

$$
\int_{D_{T}}|u|^{\alpha} \varphi_{T} d x d t \leq \frac{\alpha}{\left(\lambda_{1} \alpha-\varepsilon\right) \alpha^{\prime} \varepsilon^{\alpha^{\prime}-1}} \int_{D_{T}} \frac{\left|\square \varphi_{T}\right|^{\alpha^{\prime}}}{\varphi_{T}^{\alpha^{\prime}-1}} d x d t-\frac{\alpha}{\lambda_{1} \alpha-\varepsilon} \zeta(T) .
$$


Since $\alpha^{\prime}=\frac{\alpha}{\alpha-1}, \alpha=\frac{\alpha^{\prime}}{\alpha^{\prime}-1}$ and $\min _{0<\varepsilon<\lambda_{1} \alpha} \frac{\alpha}{\left(\lambda_{1} \alpha-\varepsilon\right) \alpha^{\prime} \varepsilon^{\alpha^{\prime}-1}}=\frac{1}{\lambda_{1}^{\alpha^{\prime}}}$, which is reached for $\varepsilon=\lambda_{1}$, then from (5.12) it follows that

$$
\int_{D_{T}}|u|^{\alpha} \varphi_{T} d x d t \leq \frac{1}{\lambda_{1}^{\alpha^{\prime}}} \int_{D_{T}} \frac{\left|\square \varphi_{T}\right|^{\alpha^{\prime}}}{\varphi_{T}^{\alpha^{\prime}-1}} d x d t-\frac{\alpha^{\prime}}{\lambda_{1}} \zeta(T)
$$

Since $\varphi_{T}:=\varphi^{0}\left(\frac{x}{T}, \frac{t}{T}\right)$ and due to (5.5), (5.6), after the change of variables $x=T x^{\prime}, t=T t^{\prime}$, it is easy to verify that

$$
\int_{D_{T}} \frac{\left|\square \varphi_{T}\right|^{\alpha^{\prime}}}{\varphi_{T}^{\alpha^{\prime}-1}} d x d t=T^{n+1-2 \alpha^{\prime}} \int_{D_{T=1}} \frac{\left|\square \varphi^{0}\right|^{\alpha^{\prime}}}{\left(\varphi^{0}\right)^{\alpha^{\prime}-1}} d x^{\prime} d t^{\prime}=T^{n+1-2 \alpha^{\prime}} æ_{0}<+\infty .
$$

Whence due to (5.7) from the inequality (5.13) we receive

$$
0 \leq \int_{D_{T}}|u|^{\alpha} \varphi_{T} d x d t \leq \frac{1}{\lambda_{1}^{\alpha^{\prime}}} T^{n+1-2 \alpha^{\prime}} æ_{0}-\frac{\alpha^{\prime}}{\lambda_{1}} \zeta(T) .
$$

Since $1<\alpha<\frac{n+1}{n-1}$ and $\alpha^{\prime}=\frac{\alpha}{\alpha-1}>1$, then $n+1-2 \alpha^{\prime}<0$ and according to $(5.6)$ we have

$$
\lim _{T \rightarrow \infty} \frac{1}{\lambda_{1}^{\alpha^{\prime}}} T^{n+1-2 \alpha^{\prime}} æ_{0}=0 .
$$

Therefore, in view of (5.9) there exists the positive number $T_{0}:=T_{0}(F)$ such that for $T>T_{0}$ the right-side of the inequality (5.14) will be negative, whereas the left-side of this inequality is nonnegative. Thus, if in conditions of Theorem 5.2 there exists a strong generalized solution of the problem (1.1), (1.2) of the class $W_{2}^{1}$ in the domain $D_{T}$, then necessarily $T \leq T_{0}$. This proves Theorem 5.2 .

The theorem below immediately follows from the proof of Theorem 5.2.

Theorem 5.3. Let $\lambda>0,1<\alpha<\frac{n+1}{n-1}$, the conditions (1.4), (1.5) be fulfilled and $F^{0} \in L_{2}\left(D_{T}\right), F^{0} \leq 0,\left\|F^{0}\right\|_{L_{2}\left(D_{T}\right)} \neq 0$. Then if $F=\mu F^{0}, g=0$, there exists the number $\mu_{0}=\mu_{0}\left(F^{0}\right)>0$ such that for $\mu>\mu_{0}$ the problem (1.1), (1.2) cannot have a strong generalized solution of the class $W_{2}^{1}$ in the domain $D_{T}$.

\section{The local solvability of the problem (1.1), (1.2)}

Below we show that despite the absence of the global solvability of the problem (1.1), (1.2) in certain cases, for example as it was shown in the previous section with violation of the condition (1.5), the problem is locally solvable in the sense of Definition 1.5 with whatever sign of the parameter $\lambda$ at the nonlinear term $f(u)$ in the equation (1.1). Below, for simplicity of consideration, we set $g=0$ in the boundary condition (1.2).

Theorem 6.1. Let $0<\alpha<\frac{n+1}{n-1}$, the condition (1.3), (1.4) be fulfilled, $g=0$, $F \in L_{2, l o c}(D)$, and $\left.F\right|_{D_{T}} \in L_{2}\left(D_{T}\right)$, for every $T>0$. Then the problem (1.1), (1.2) is locally solvable in the class $W_{2}^{1}$, i.e. there exists the number $T_{0}=$ $T_{0}(F)>0$ such that for $T<T_{0}$ the problem has a strong generalized solution of the class $W_{2}^{1}$ in the domain $D_{T}$. 
Proof. In the conditions of Theorem 6.1, according to Remark 3.1, the function $u \in \stackrel{0}{W}_{2}^{1}\left(D_{T}, S_{T}\right):=\left\{v \in W_{2}^{1}\left(D_{T}\right):\left.v\right|_{S_{T}}=0\right\}$ is a strong generalized solution of the problem (1.1), (1.2) with $g=0$ of the class $W_{2}^{1}$ in the domain $D_{T}$ if and only if $u$ is a solution of the functional equation (3.12), i.e.

$$
u=A_{0} u:=-L_{01}^{-1}\left(K_{0} u\right)+L_{01}^{-1}(F) .
$$

in the space $\stackrel{0}{W} \underset{2}{1}\left(D_{T}\right)$. Here $L_{01}^{-1}: L_{2}\left(D_{T}\right) \rightarrow \stackrel{0}{W} \underset{2}{1}\left(D_{T}, S_{T}\right)$ is the linear continuous operator with norm satisfying the first inequality (3.10), while the operator $K_{0}: \stackrel{0}{W} \underset{2}{1}\left(D_{T}, S_{T}\right) \rightarrow L_{2}\left(D_{T}\right)$, acting according to formula

$$
K_{0} u=-\lambda f(u),
$$

is continuous and compact together with the operator $A_{0}: \stackrel{0}{W} \underset{2}{1}\left(D_{T}, S_{T}\right) \rightarrow$ $\stackrel{0}{W} \underset{2}{1}\left(D_{T}, S_{T}\right)$ from (6.1). Therefore, due to the Sobolev theorem [32, p. 270] for the solvability of the equation (6.1) in the space ${ }_{W}^{W}{ }_{2}^{1}\left(D_{T}, S_{T}\right)$ it suffices to show that for any fixed number $R>0$ and sufficiently small positive $T$ the operator $A_{0}$ transforms the closed convex ball $B(0, R):=\left\{u \in \stackrel{0}{W}_{2}^{1}\left(D_{T}, S_{T}\right)\right.$ : $\left.\|u\|_{W_{2}^{\frac{1}{2}}\left(D_{T}, S_{T}\right)} \leq R\right\}$ with a center in null element and the radius $R$ into itself in the Hilbert space ${ }_{W}^{W} \underset{2}{1}\left(D_{T}, S_{T}\right)$. With this purpose let us estimate the value of $\left\|A_{0} u\right\|_{W_{2}^{\frac{1}{2}\left(D_{T}, S_{T}\right)}}$ for $u \in \stackrel{0}{W} \underset{2}{1}\left(D_{T}, S_{T}\right)$.

First let us put that $1<\alpha<\frac{n+1}{n-1}$. If $u \in \stackrel{0}{W}_{2}^{1}\left(D_{T}, S_{T}\right)$, then we denote by $\tilde{u}$ the function which is the even continuation of the function $u$ through the plane $t=T$ into the domain $D_{T}^{*}$, symmetrical to $D_{T}$ with respect to the same plane $t=T$, i.e.

$$
\tilde{u}= \begin{cases}u(x, t), & (x, t) \in D_{T} ; \\ u(x, 2 T-t), & (x, t) \in D_{T}^{*},\end{cases}
$$

and $\tilde{u}(x, t)=u(x, t)$ for $t=T$ in the sense of the trace theory. Obviously, $\tilde{u} \in \stackrel{0}{W}_{2}^{1}\left(\tilde{D}_{T}\right):=\left\{v \in W_{2}^{1}\left(\tilde{D}_{T}\right):\left.v\right|_{\partial \tilde{D}_{T}}=0\right\}$, where $\tilde{D}_{T}=D_{T} \cup \Omega_{T} \cup$ $D_{T}^{*}, \Omega_{T}:=D \cap\{t=T\}$.

Using the inequality [33, p. 258]

$$
\int_{\Omega}|v| d \Omega \leq(m e s \Omega)^{1-\frac{1}{p}}\|v\|_{p, \Omega}, \quad p \geq 1,
$$

and taking into account the equalities

$$
\|\tilde{u}\|_{L_{p}\left(\tilde{D}_{T}\right)}^{p}=2\|u\|_{L_{p}\left(D_{T}\right)}^{p}, \quad\|\tilde{u}\|_{W_{2}^{\prime}\left(\tilde{D}_{T}\right)}^{2}=2\|u\|_{W_{2}^{\prime}\left(D_{T}, S_{T}\right)}^{2},
$$

from the well-known multiplicative inequality [25, p. 78]

$$
\begin{aligned}
& \|v\|_{p, \Omega} \leq \beta\left\|\nabla_{x, t} v\right\|_{m, \Omega}^{\tilde{\alpha}}\|v\|_{r, \Omega}^{1-\tilde{\alpha}} \quad \forall v \in \stackrel{0}{W}_{2}^{1}(\Omega), \Omega \subset \mathbb{R}^{n+1} \\
& \nabla_{x, t}=\left(\frac{\partial}{\partial x_{1}}, \ldots, \frac{\partial}{\partial x_{n}}, \frac{\partial}{\partial t}\right), \quad \tilde{\alpha}=\left(\frac{1}{r}-\frac{1}{p}\right)\left(\frac{1}{r}-\frac{1}{\tilde{m}}\right)^{-1}, \quad \tilde{m}=\frac{(n+1) m}{n+1-m},
\end{aligned}
$$


for $\Omega=\tilde{D}_{T} \in \mathbb{R}^{n+1}, v=\tilde{u}, r=1, m=2$ and $1<p \leq \frac{2(n+1)}{n-1}$, where $\beta=$ const $>0$ does not depend on $v$ and $T$, we obtain the inequality

$$
\|u\|_{L_{p}\left(D_{T}\right)} \leq c_{0}\left(\operatorname{mes} D_{T}\right)^{\frac{1}{p}+\frac{1}{n+1}-\frac{1}{2}}\|u\|_{W_{\frac{1}{2}}^{1}\left(D_{T}, S_{T}\right)} \quad \forall u \in \stackrel{0}{W} \underset{2}{1}\left(D_{T}, S_{T}\right),
$$

where $c_{0}=$ const $>0$ is independent of $u$ and $T$.

Taking into account that mess $D_{T}=\frac{\omega_{n}}{n+1} T^{n+1}$, where $\omega_{n}$ is the volume of the unit ball in $\mathbb{R}^{n}$, from (6.3) for $p=2 \alpha$ we obtain

$$
\|u\|_{L_{2 \alpha}\left(D_{T}\right)} \leq C_{T}\|u\|_{W_{2}^{0}\left(D_{T}, S_{T}\right)} \quad \forall u \in \stackrel{0}{W} \underset{2}{1}\left(D_{T}, S_{T}\right),
$$

where

$$
C_{T}=c_{0}\left(\frac{\omega_{n}}{n+1}\right)^{\alpha_{1}} T^{(n+1) \alpha_{1}}, \quad \alpha_{1}=\frac{1}{2 \alpha}+\frac{1}{n+1}-\frac{1}{2} .
$$

Since $\alpha<\frac{n+1}{n-1}$, then $\frac{1}{2 \alpha}+\frac{1}{n+1}-\frac{1}{2}>0$ and in view of (6.4) and (6.5), for every $u \in \stackrel{0}{W} \underset{2}{1}\left(D_{T}, S_{T}\right)$, we have

$$
\|u\|_{L_{2 \alpha}\left(D_{T}\right)} \leq C_{T_{1}}\|u\|_{W_{\frac{1}{2}\left(D_{T}, S_{T}\right)}^{0}} \quad \forall T \leq T_{1},
$$

where $T_{1}$ is a certain fixed positive number.

By virtue of (1.4), (6.2) and (6.6) for every $u \in \stackrel{0}{W} \underset{2}{1}\left(D_{T}, S_{T}\right)$ and $T \leq T_{1}$ we have

$$
\begin{aligned}
& \left\|K_{0} u\right\|_{L_{2}\left(D_{T}\right)}^{2} \leq 2|\lambda|^{2} \int_{D_{T}}\left(M_{1}^{2}+M_{2}^{2}|u|^{2 \alpha}\right) d x d t \leq 2|\lambda|^{2} M_{1}^{2} \text { mes } D_{T} \\
& +2|\lambda|^{2} M_{2}^{2}\|u\|_{L_{2 \alpha}\left(D_{T}\right)}^{2 \alpha} \leq 2|\lambda|^{2} M_{1}^{2} \text { mes } D_{T}+2|\lambda|^{2} M_{2}^{2} C_{T_{1}}^{2 \alpha}\|u\|_{W_{2}^{\prime}\left(D_{T}, S_{T}\right)}^{2 \alpha}
\end{aligned}
$$

and therefore

$$
\left\|K_{0} u\right\|_{L_{2}\left(D_{T}\right)} \leq \sqrt{2}|\lambda| M_{1} \sqrt{m e s D_{T}}+\sqrt{2}|\lambda| M_{2} C_{T_{1}}^{\alpha}\|u\|_{W_{2}^{1}\left(D_{T}, S_{T}\right)}^{\alpha} .
$$

Further, from (3.4), (3.10), (6.1) and (6.7) it follows that

$$
\begin{aligned}
\left\|A_{0} u\right\|_{W_{2}^{1}\left(D_{T}, S_{T}\right)} \leq\left\|L_{01}^{-1}\right\|_{L_{2}\left(D_{T}\right) \rightarrow W_{2}^{1}\left(D_{T}, S_{T}\right)}\left\|K_{0} u\right\|_{L_{2}\left(D_{T}\right)} \\
\quad+\left\|L_{01}^{-1}\right\|_{L_{2}\left(D_{T}\right) \rightarrow W_{2}^{1}\left(D_{T}, S_{T}\right)}\|F\|_{L_{2}\left(D_{T}\right)} \\
\leq c\left[\sqrt{2}|\lambda| M_{1} \sqrt{m e s D_{T_{1}}}+\sqrt{2}|\lambda| M_{2} C_{T_{1}}^{\alpha}\|u\|_{W_{2}^{1}\left(D_{T}, S_{T}\right)}^{\alpha}\right. \\
\left.\quad+\|F\|_{L_{2}\left(D_{T_{1}}\right)}\right] \\
\leq \sqrt{T}\left(M_{0}+2\right)^{\frac{1}{2}} \exp \left(T_{1}+\frac{1}{2}\right) T_{1}\left[\sqrt{2}|\lambda| M_{1} \sqrt{m e s D_{T_{1}}}\right. \\
\left.\quad+\sqrt{2}|\lambda| M_{2} C_{T_{1}}^{\alpha}\|u\|_{W}^{\alpha} \underset{\frac{1}{2}\left(D_{T}, S_{T}\right)}{\alpha}+\|F\|_{L_{2}\left(D_{T_{1}}\right)}\right], \\
\forall T \leq T_{1}, \quad \forall u \in W_{2}^{1}\left(D_{T}, S_{T}\right) .
\end{aligned}
$$


Since the right-side of the inequality (6.8) contains $\sqrt{T}$ as a factor, which tends to zero for $T \rightarrow 0$, then there exists the positive number $T_{0} \leq T_{1}$ such that for $T<T_{0}$ and $\|u\|_{W_{2}^{\frac{1}{2}\left(D_{T}, S_{T}\right)}} \leq R$, due to (6.8), we have $\left\|A_{0} u\right\|_{W_{2}^{1}\left(D_{T}\right)} \leq$ $R$, i.e. the operator $A_{0}: \stackrel{0}{W} \underset{2}{1}\left(D_{T}, S_{T}\right) \rightarrow \stackrel{0}{W} \underset{2}{1}\left(D_{T}, S_{T}\right)$ from (6.1) transforms the ball $B(0, R)$ into itself.

The case when $0<\alpha \leq 1$ can be investigated in the same way if we use beforehand the known inequality [33, p. 258]

$$
\|v\|_{L_{\alpha}(G)} \leq(\operatorname{mes} G)^{\frac{\alpha_{1}-\alpha}{\alpha_{1} \alpha}}\|v\|_{L_{\alpha_{1}}(G)}
$$

where $\alpha_{1}$ is a certain fixed number from $\left(1, \frac{n+1}{n-1}\right)$. This proves Theorem 6.1 .

\section{References}

[1] Sobolev, S.L.: Some new problems of the theory of partial differential equations of hyperbolic type (Russian). Mat. Sb. 11(53)(3), 155-200 (1942)

[2] Kharibegashvili, S.S.: On the existence or absence of global solutions for the multidimensional version of the second Darboux problem for some nonlinear hyperbolic equations. Differ. Equ. 43(3), 402-416 (2007). Translated from Differentsial'nye Uravneniya 43(3), 388-401 (2007)

[3] Kharibegashvili, S.: Boundary value problems for some classes of nonlinear wave equations. Mem. Differ. Equ. Math. Phys. 46, 1-114 (2009)

[4] Bitsadze, A.V.: Some Classes of Partial Differential Equations (Russian). Izdat. "Nauka", Moscow (1981)

[5] Jorgens, K.: Das Anfangswertproblem im Grossen fur eine Klasse nichtlinearer Wellengleichungen. Math. Z. 77, 295-308 (1961)

[6] Segal, I.E.: The global Cauchy problem for a relativistic scalar field with power interaction. Bull. Soc. Math. France 91, 129-135 (1963)

[7] Lions, I.L.: Quelques méthods de résolution des problèmes aux limites non linéaires. Dunod, Gauthier-Villars, Paris (1969)

[8] Levine, H.A.: Instability and nonexistence of global solutions to nonlinear wave equations of the form $P u_{t t}=-A u+F(u)$. Trans. Am. Math. Soc. 192, 1-21 (1974)

[9] John, F.: Blow-up of solutions of nonlinear wave equations in three space dimensions. Manuscr. Math. 28(1-3), 235-268 (1979)

[10] Kato, T.: Blow-up of solutions of some nonlinear hyperbolic equations. Comm. Pure Appl. Math. 33(4), 501-505 (1980)

[11] Sideris, T.C.: Nonexistence of global solutions to semilinear wave equations in high dimensions. J. Differ. Equ. 52(3), 378-406 (1984) 
[12] Hörmander, L.: Lectures on nonlinear hyperbolic differential equations. In: Mathématics \& Applications (Berlin), vol. 26. Springer-Verlag, Berlin (1997)

[13] Levine, H.A., Park, S.R.: Global existence and global nonexistence of solutions of the Cauchy Problem for a nonlinearly damped wave equation. J. Math. Anal. Appl. 228, 181-205 (1998)

[14] Mitidieri, E., Pohozaev, S.I.: A priori estimates and the absence of solutions of nonlinear partial differential equations and inequalities (Russian). Trudy Mat. Inst. Steklova, 234, 1-384 (2001); English transl.: Proc. Steklov Inst. Math. 234(3), 1-362 (2001)

[15] Ikehata, R., Tanizawa, K.: Global existence of solutions for semilinear damped wave equations in $\mathbb{R}^{n}$ with noncompactly supported initial data. Nonlinear Anal. 61, 1189-1208 (2005)

[16] Todorova, G., Vitillaro, E.: Blow-up for nonlinear dissipative wave equations in $\mathbb{R}^{n}$. J. Math. Anal. Appl. 303(1), 242-257 (2005)

[17] Kharibegashvili, S.: On the existence or the absence of global solutions of the Cauchy characteristic problem for some nonlinear hyperbolic equations. J. Bound. Value Probl. 2005(3), 359-376 (2005)

[18] Kharibegashvili, S.: On the nonexistence of global solutions of the characteristic Cauchy problem for a nonlinear wave equation in a conical domain. Differ. Equ. 42(2), 279-290 (2006). Translated from Differential'nye Uravneniya, 42(2), 261-271 (2006)

[19] Kharibegashvili, S.: Some multidimensional problems for hyperbolic partial differential equations and systems. Mem. Differ. Equ. Math. Phys. 37, 1-136 (2006)

[20] Kharibegashvili, S.: On the solvability of one multidimensional version of the first Darboux problem for some nonlinear wave equations. Nonlinear Anal. 68, 912-924 (2008)

[21] Kharibegashvili, S.: On the solvability of one multidimensional version of the Cauchy characteristic problem for a nonlinear equation with iterated wave operator in the principal part. J. Math. Anal. Appl. 338, 71-84 (2008)

[22] Yao, P.-F.: Global smooth solutions for the quasilinear wave equations with boundary dissipation. J. Differ. Equ. 241, 62-93 (2007)

[23] Zhou, Y.: Global existence and nonexistence for a nonlinear wave equation with damping and source term. Math. Nachr. 278(11), 1341-1358 (2008)

[24] Kenig, C.E., Merle, F.: Global well-posedness, scattering and blow-up for the energy-critical focusing non-linear wave equation. Acta Math. 201, 147$212(2008)$

[25] Ladyzhenskaya, O.A.: Boundary Value Problems of Mathematical Physics (Russian). Izdat. "Nauka", Moscow (1973)

[26] Krasnosel'skii, M.A., Zabreiko, P.P., Pustyl'nik, E.I., Sobolevskii, P.E.: Integral operators in spaces of summable functions (Russian). Izdat. "Nauka", Moscow (1966) 
[27] Kufner, A., Fuchik, S.: Nonlinear differential equations. (Translated into Russian) Izdat. "Nauka", Moscow (1988)

[28] Henry, D.: Geometric theory of semilinear parabolic equations. (Translated into Russian) Izdat. "Mir", Moscow (1985)

[29] Kondrat'ev, V.A.: Boundary value problems for elliptic equations in domains with conical or angular points (Russian). Trudy Moskov. Mat. Obshch. 16, 209292 (1967)

[30] Kharibegashvili, S.: Goursat and Darboux type problems for linear hyperbolic partial differential equations and systems. Mem. Differ. Equ. Math. Phys. 4, $1-127$ (1995)

[31] Kondrat'ev, V.A.: On solvability of the first boundary value problem for strongly elliptic equations (Russian). Trudy Moskov. Mat. Obshch. 16, 293-318 (1967)

[32] Trenogin, V.A.: Functional analysis (Russian). Izdat. "Nauka", Moscow (1993)

[33] Vulikh, B.Z.: Concise course of the theory of function of a real variable (Russian). Izdat. "Nauka", Moscow (1973)

S. Kharibegashvili

A. Razmadze Mathematical Institute, 1, M. Aleksidze St., 0193 Tbilisi,

Georgia

e-mail: kharibegashvili@yahoo.com

S. Kharibegashvili

Department of Mathematics,

Georgian Technical University,

77, M. Kostava Str.,

0175 Tbilisi,

Georgia

B. Midodashvili

I. Javakhishvili Tbilisi State University,

2, University St.,

0143 Tbilisi,

Georgia

e-mail: bidmid@hotmail.com

Received: 17 February 2010.

Revised: 04 July 2010.

Accepted: 02 September 2010. 